\title{
Tabagismo: a percepção dos fumantes em um grupo de educação em saúde
}

\author{
Smoking: the perception of smokers on a health education group \\ Tabaquismo: la percepción de los fumadores en un grupo de educación en la salud \\ Noely Soares Veloso ${ }^{*}$, Carlos Alberto Quintão Rodrigues², Maísa Tavares de Souza Leite ${ }^{3}$, Julimary Larissa \\ Mendes Ottoni ${ }^{4}$, Geane Christie do Carmo Veloso ${ }^{5}$, Rosiane Mendes Rodrigues ${ }^{6}$, Gabriela Alencar Bandeira ${ }^{7}$
}

Palavras-chave: Tabagismo Atenção Primária Educação em Saúde Fatores de Risco Saúde da Família

Keywords: Smoking Primary Care Health Education

Risk Factors

Family Health

Palabras clave: Tabaquismo Atención Primaria Educación en Salud Factores de Riesgo Salud de la Familia

\section{Resumo}

Estudo qualitativo que objetivou compreender o significado dos grupos antitabagismo desenvolvidos em uma unidade de Saúde da Família do município de Montes Claros, em Minas Gerais. Utilizou-se como referencial teórico-metodológico a pesquisa-ação. Os sujeitos foram usuários tabagistas que vivenciaram as sessões do grupo de educação em saúde. Os instrumentos para coleta de dados foram: observação não-participante, observação participante e entrevistas semiestruturadas. Os dados foram submetidos à técnica da análise do discurso com identificação de duas categorias: 0 significado do tabagismo e o significado dos grupos de apoio antitabagismo na percepção dos sujeitos participantes do grupo. Emergiram as ideias e os sentimentos dos fumantes, bem como a representatividade do cigarro para os sujeitos. 0 estudo demonstrou a relevância dos grupos de apoio, durante o processo de cessação do tabagismo, e a necessidade de melhor capacitação profissional e estruturação dos serviços para o enfrentamento do problema. Este estudo também se constituiu numa forma de divulgação do tratamento do tabagismo na rede pública de saúde e do seu impacto na população beneficiada, servindo de parâmetro e estímulo a outros cenários.

\section{Abstract}

This is a qualitative study which aimed to understanding the meaning of anti-smoking groups developed in a unit of Family Health Care in the city of Montes Claros, in Minas Gerais State. The action-research was used as a theoretical and methodological reference. The subjects were smokers who experienced group sessions on health education. The data collection tools were: the non-participant observation, the participant observation, and the semi-structured interviews. The collected data were submitted to the technique of discourse analysis with the identification of two categories: the meaning of smoking and the meaning of anti-smoking support groups in the perception of the subjects who were part of the group. Smokers expressed ideas and feelings as well as the representation of the cigarette. This study showed the relevance of support groups during the process of quitting smoking, and the need of better professional training and service structure in order to face the problem. This study was also a way to promote the anti-smoking treatment in the public health system and its impact on the population who had this benefit, being a parameter and encouragement for other scenarios.

\section{Resumen}

Estudio cualitativo que tuvo como objetivo la comprensión del significado de los grupos contra el tabaquismo, desarrollados en una unidad de la Salud de la Familia del municipio de Montes Claros, en Minas Gerais. Ha sido utilizado como material referencial teóricometodológico la investigación-acción. Los individuos fueron usuarios de tabaco que participaron de las sesiones del grupo educación en

\footnotetext{
'Universidade Estadual de Montes Claros (Unimontes). noely.veloso@yahoo.com.br

${ }^{2}$ PET-saúde Universidade Estadual de Montes Claros (Unimontes). carlosquintao@hotmail.com

${ }^{3}$ Departamento de Enfermagem da Universidade Estadual de Montes Claros (Unimontes); PET-saúde Universidade Estadual de Montes Claros (Unimontes). mtsiv@terra.com.br

${ }^{4}$ ESF Vila Telma; PET-saúde Universidade Estadual de Montes Claros (Unimontes). julyottoni@yahoo.com.br

5Universidade Estadual de Montes Claros (Unimontes). drachristie@hotmail.com

6Universidade Estadual de Montes Claros (Unimontes). rosemendesrodrigues@yahoo.com.br

${ }^{7}$ Universidade Estadual de Montes Claros (Unimontes). gabriela.bandeira02@hotmail.com

*Autor correspondente.

Fonte de financiamento: nenhuma.

Conflito de interesses: declararam não haver.

Recebido em: 30/03/2011

Aprovado em: 28/10/2011
} 
la salud. Los instrumentos para la colecta de datos fueron la observación no participante, la observación participante y las entrevistas semiestructuradas. Los datos han sido sometidos a la técnica del análisis de discurso con la identificación de dos categorías: el significado del tabaquismo y el significado de los grupos de apoyo antitabaquismo en la percepción de los participantes del grupo. Surgieron las ideas y sentimientos de los fumadores, bien como la representatividad del cigarrillo para los individuos. El estudio ha demostrado la pertinencia de los grupos de apoyo, durante el proceso de cese del tabaquismo, y la necesidad de una mejor formación profesional y estructuración de los servicios para el enfrentamiento del problema. Este estudio también se constituye en una forma de divulgación del tratamiento del tabaquismo en la red pública de la salud y de su impacto en la población beneficiada, sirviendo de parámetro y estímulo a otros escenarios.

\section{Introdução}

De acordo com a Organização Mundial de Saúde (OMS), o uso do tabaco é responsável por 4,9 milhões de mortes ao ano, sendo a maior causa de morte evitável identificada. Ainda, segundo a OMS, ao ano, quatro milhões de homens e um milhão de mulheres morrem devido aos malefícios do fumo e, caso a expansão permaneça, esses números podem chegar a dez milhões de mortes anuais, em $2020^{1}$. Estima-se que, em 2030, oito milhões de pessoas morrerão por doenças ligadas ao tabaco; destas, mais de $80 \%$ ocorrerão em países de baixa e média rendas ${ }^{2}$.

O Ministério da Saúde enfatiza que os prejuízos à saúde pelo uso dos derivados do tabaco não se limitam ao câncer de pulmão e a outros cânceres, mas também incluem o enfisema; a bronquite crônica; as doenças cardiovasculares, como o infarto do miocárdio; e os acidentes vasculares encefálicos, grandes causas de morbimortalidade no Brasil, além do envelhecimento prematuro da pele, entre outras doenças ${ }^{3}$.

O desgaste econômico com o tabagismo é muito grande, envolvendo indivíduos, serviços e a sociedade como um todo. Há gastos públicos com o tratamento das doenças associadas ao tabagismo e com a diminuição de produtividade dos fumantes acometidos por essas doenças. $\mathrm{O}$ gasto familiar se compromete com o hábito de fumar (10\% do orçamento global em famílias pobres), e o uso de tabaco exacerba a pobreza e a falta de escolaridade ${ }^{4}$.

A Atenção Primária, enquanto cenário maior de promoção da saúde, tem produzido iniciativas relevantes no combate ao tabagismo, porém ainda com produção científica escassa relatada. Considerando o fator da interdisciplinaridade, ainda poucos resultados são encontrados. Como afirma Oliveira ${ }^{5}$, percebe-se que a pesquisa interdisciplinar em saúde é fundamental para a progressiva construção de um modelo, no qual a saúde coletiva seja abordada de maneira profunda. Dessa forma, busca-se uma integração e a construção do conhecimento, articulando o serviço e o ensino.

Esta pesquisa teve como objetivo a compreensão do significado do tabagismo para usuários de uma unidade de Saúde da Família (SF), bem como o significado dos grupos antitabagismo, que foram implementados para esses mesmos indivíduos envolvidos, com integração entre professores e estudantes das áreas da medicina, enfermagem e odontologia, tendo em vista a integralidade do cuidado, a visão multiprofissional e o desenvolvimento de ações interdisciplinares.

O presente estudo visa ainda colaborar para a divulgação do tratamento do tabagismo na rede pública de saúde - Sistema Único de Saúde (SUS), e para o impacto dessa estratégia na população beneficiada, o que servirá de parâmetro e estímulo a outros cenários.

\section{Métodos}

Trata-se de um estudo descritivo com abordagem qualitativa, cujo referencial teórico-metodológico foi a pesquisaação. Essa metodologia foi escolhida por ser adequada a um problema complexo, de natureza social e cultural, como o tabagismo.

De acordo com Thiollent ${ }^{6}$, a pesquisa-ação é “[...] um tipo de pesquisa social com base empírica que é concebida e realizada em estreita associação com uma ação ou com a resolução de um problema coletivo e no qual os pesquisadores e os participantes representativos da situação ou do problema estão envolvidos de modo cooperativo ou participativo".

Ainda, segundo o mesmo autor, para uma pesquisa ser qualificada como pesquisa-ação, deve haver ativa participação por parte das pessoas ou grupos implicados no problema sob observação. Além disso, é preciso que a ação seja uma ação não-trivial, ou seja, uma ação problemática, que merece investigação para ser elaborada e conduzida.

$\mathrm{O}$ cenário deste estudo foi a área de abrangência de uma unidade de SF em Montes Claros, no estado de Minas Gerais, de maio de 2009 a fevereiro de 2010.

Os sujeitos do estudo foram usuários tabagistas cadastrados na referida unidade, que manifestaram espontaneamente o desejo de parar de fumar ou foram identificados pelos agentes comunitários de saúde. Foram utilizados os seguintes critérios de inclusão: fazer parte da área de abrangência daquela unidade de SF; encontrar-se no chamado 
"período de ação", ou seja, com desejo de cessação do tabagismo dentro de 30 dias; e estar disponível para frequentar as reuniões.

Os aspectos éticos desta pesquisa foram considerados de acordo com a Resolução 196 de 10/10/1996, da Comissão Nacional de Ética em Pesquisa do Ministério da Saúde, que estipula normas éticas regulamentadoras de pesquisas envolvendo seres humanos. Desse modo, este projeto foi submetido à apreciação e aprovação do Comitê de Ética em Pesquisa (CEP - no 1.521/09) da Universidade Estadual de Montes Claros (UNIMONTES), antes da coleta dos dados.

Foram utilizados como instrumentos para coleta de dados: observação não-participante (primeira sessão), observação participante (demais sessões) e entrevistas semiestruturadas.

No decorrer deste estudo, foram realizadas duas entrevistas semiestruturadas após o segundo e quarto encontros, de duração de aproximadamente 40 minutos, com o intuito de responder às seguintes questões norteadoras: "Qual o significado do tabagismo para você?" e "Qual o significado do grupo para você?". Novas questões foram utilizadas quando houve a necessidade de esclarecer as reais representações para os sujeitos.

A análise foi feita em etapas, de forma gradativa e qualitativa, com a avaliação das diferentes fases, por meio da análise do discurso dos participantes. Durante as etapas, houve participação multiprofissional (medicina, enfermagem e odontologia) que, conforme defende Marques ${ }^{7}$, favorece a discussão e a articulação de saberes. Acadêmicos destes três cursos da UNIMONTES também participaram ativamente nesse processo.

A análise do discurso tem como objetivo básico fazer uma reflexão geral sobre as condições de produção e apreensão da significação das falas. Dessa forma, é fundamental a relação entre a linguagem e o contexto de sua produção ${ }^{8}$. Para se alcançar a interpretação dos sujeitos em uma dimensão mais ampla, foi necessária, além da análise das falas, a observação constante dos gestos, das expressões corporais e dos comportamentos. Objetivando a fidedignidade dos relatos, foram utilizados dispositivos de gravação de áudio e anotação escrita.

No decorrer da análise, foram identificadas duas categorias: o significado do tabagismo na percepção dos sujeitos e o significado dos grupos antitabagismo para os mesmos.

Visando manter o anonimato, as falas foram identificadas por códigos que distinguem o participante e o momento da coleta do dado. Por exemplo, P4 E1: participante número 4 na entrevista de número 1; ou P5 S2: participante número cinco na sessão de número 2 .
O presente trabalho recebeu apoio logístico e financeiro do Programa de Educação pelo Trabalho para a Saúde (PET- Saúde), que firmou convênio com a referida universidade.

\section{Resultados e Discussão}

Diante da necessidade de uma estratégia de combate ao tabagismo em uma unidade de SF, adotou-se uma sequência de ações sugeridas pelo Ministério da Saúde e pelo Instituto Nacional de Câncer (INCA) - Abordagem e Tratamento do Fumante ${ }^{9}$, que obedeceu aos seguintes passos:

- Capacitação dos profissionais das áreas de odontologia, medicina e enfermagem, assim como dos estudantes das respectivas áreas, para conhecimento do programa e da abordagem cognitivo-comportamental.

- Triagem com o objetivo de identificar as condições clínicas dos interessados, história tabagística e o grau de motivação.

- Sessões com os participantes definidos na fase anterior, sendo quatro semanais, duas quinzenais e mensais de manutenção no decorrer do ano, todas com participação interdisciplinar (com professores e estudantes dos cursos de enfermagem, medicina e odontologia). As sessões obedeceram à sequência sugerida pelo Ministério da Saúde, sendo as quatro primeiras com as seguintes temáticas: entender por que se fuma e como isso afeta a saúde; os primeiros dias sem fumar; como vencer os obstáculos para permanecer sem fumar; e os benefícios obtidos após parar de fumar. Em todas as sessões, procurou-se dar ênfase à atenção individual.

\section{Algumas considerações a respeito do grupo}

O grupo se constituiu de usuários, em sua maioria, do gênero feminino (sete mulheres e dois homens), todos alfabetizados, com início do uso do tabaco variando entre 13 e 25 anos de idade. O número de participantes se definiu pela demanda espontânea e pelo preenchimento dos critérios de inclusão. No questionário de triagem, verificou-se que sete dos participantes já haviam tentado interromper o tabagismo por uma a três vezes. Dois participantes já haviam tentado a cessação por mais de três episódios. Ainda foi verificado, pelo Teste de Fagerstöm ${ }^{10}$, que a maioria dos participantes apresentava grau de dependência de médio a elevado. 


\section{Significado do tabagismo na percepção dos participantes do grupo}

Com o passar do tempo, o tabaco já apresentou inúmeras conotações culturalmente positivas, reforçadas pela mídia e convenientes para uma sociedade produtora de uma carga progressivamente alta de tensão, competitividade e imediatismo. O cigarro já significou instrumento de poder, sedução, intelectualidade e rebeldia. Atualmente, com todos os conhecimentos a respeito do seu uso, persiste frequente no cotidiano das pessoas, causando tantos danos à população geral. Que significado, então, esse tão relevante agravo à saúde tem para os indivíduos?

No cenário em que foi realizado este estudo, os sujeitos demonstraram um significado ambíguo e paradoxal para o tabaco. Ao mesmo tempo eles o consideram uma fonte de alívio e de apoio, e, de outro lado, "destruição".

Parte dos entrevistados relatou uma forte relação do tabagismo como uma forma de alívio para as tensões e situações de estresse enfrentadas no decorrer de suas vidas:

O cigarro era um amigo. Ele me acalmava quando estava nervosa. (P1 E1)

Tudo na minha vida (...). Resolve meu nervosismo (...) (P6 E1)

É um prazer, um apoio emocional. (P8 E1)

Pensava que quando fumava resolvia os problemas. (P4 S3)

Isso confirma as reflexões de um estudo ${ }^{11}$ recente direcionado ao tabagismo feminino, em que o cigarro foi considerado um suporte para momentos de intenso sofrimento. Essa ligação entre o uso do tabaco e a ansiedade também confirma a pesquisa realizada por Malbergier e Oliveira ${ }^{12}$, que verificaram maior incidência e prevalência de doenças mentais entre os fumantes.

Por outro lado, outros usuários sinalizaram uma ambivalência em relação ao tabaco, com manifestação ao mesmo tempo de prazer e raiva direcionadas ao uso. Alguns relataram sintomas físicos e insatisfação:

Muita raiva, nervoso, mal-estar, dor de cabeça. (P4 E1)

Falsa impressão de prazer, alegria falsa. Achava que não fazia mal, mas causa dor no estômago, perda de cabelo, dente, peso (...) envelhecimento precoce. (P3 E1)

Muita dificuldade na minha vida. Cansaço, dor nos ossos. (P7 E1)

Escravidão, dependência mesmo. (P9 E1)

Assim, percebe-se uma dupla conotação atribuída ao tabagismo, que já havia sido percebida em outros estudos ${ }^{11}$ : o cigarro é lembrado de forma contraditória, é amigo, dá apoio, mas também é inimigo e leva à morte.

Focchi et al. ${ }^{13}$ afirmam que lidar com dependência química é tarefa árdua, como "enxugar gelo". Observou-se também na fala dos participantes o já conhecido caráter tirânico da dependência do tabaco, considerada uma das mais difíceis de romper:

A necessidade do fumo é igual à necessidade de comer. (P5 S1) Não sei explicar, é uma vontade que me deixa louca. (P5 S1)

Já tentei parar, mas não consigo. Consegui diminuir, mas parar não. (P9 S1)

Acho bom demais. Os vizinhos fumam e de longe cheira. Fiquei um ano sem fumar, mas tinha dias que eu ficava louca (...).

(P5 S2)

Ficou evidente a dificuldade de rompimento com a dependência do tabaco, com enorme disparidade entre o querer parar e a ação de fato. Vários fatores estão envolvidos com o abandono desse vício, sendo que a motivação parece ser o principal. A motivação, no entanto, sofre influência de muitas variáveis, tais como contexto social, familiar, apoio profissional e até espiritual ${ }^{14}$. Uma das participantes, por exemplo, relacionou explicitamente a sua recaída com a enfermidade grave do marido.

Deve ser considerado o contexto econômico e social dos entrevistados, que constitui outro fator fragilizador em suas situações. Estudos apontam maior prevalência do tabagismo em populações menos favorecidas ${ }^{15}$, e é possível imaginar o quanto pior é o impacto dessa dependência na vida de pessoas com baixa renda. O cigarro teve ainda o significado de válvula de escape, fuga e até revolta:

O nervosismo, os problemas da vida(...) desconto tudo no cigarro. (P6 S1)

O que é "tudo"? Os problemas, as limitações, as decepções, as oportunidades perdidas, o sonho interrompido, as insatisfações, as consequências de uma sociedade desigual ou de uma psique perturbada; tudo isso pode significar o uso do tabaco. Então, se dessa forma tudo é descontado no cigarro, em quem esse amigoinimigo vai descontar?

O tabagismo ganha assim um significado paradoxal para seus usuários, um instrumento de prazer, alívio e suporte; e, do outro lado da balança, uma fonte impiedosa de dano social e humano que precisa e deve ser enfrentada. 


\section{Significado dos grupos antitabagismo para os sujeitos}

A presença nos grupos de apoio foi positiva na visão dos participantes, que os consideraram um suporte a mais, além do comportamento da família e de amigos:

O grupo foi muito importante. Foi maravilhoso. É algo fundamental, tem que continuar(...). Acho que tem mais pessoas que precisam participar. (P3 E2)

Achei o trabalho maravilhoso, porque a experiência deu certo. Parei há 15 dias e não fumo mais. Lembro do cigarro, mas não sinto vontade. Sinto um pouco de falta de apetite. (P4 S3)

O grupo é muito importante. Conversar com as outras pessoas dá mais força para largar o vício. Para mim, o grupo foi muito importante, ajudou muito. (P4 E2)

Foi muito bom. Ajuda muito a gente. Foi bom para conbecer as pessoas e saber que há problemas maiores que o nosso. Mas acharia melhor pelo menos dois meses toda semana para dar mais força(...), quando fica muito tempo a gente acaba voltando. (P1 E2)

O grupo foi bom. Quando estava na reunião, parecia que eu tinha mais força. Eu tinha mais firmeza. (P5 E2)

Ajudou-me a diminuir. Vi os exemplos das pessoas. Vi que é difícil parar, mas que é possivel. É preciso se reestruturar para parar. (P9 E2)

Os depoimentos confirmam o significado de apoio imbuído nos grupos e ratificam o que já foi observado por Echer e Barreto ${ }^{14}$ : o grupo tem um sentido especial, porque os integrantes percebem que vivenciam um problema em comum e sabem que os demais entendem e enfrentam as mesmas dificuldades. Além disso, o grupo apresenta também caminhos e orientações, e propicia o reforço da vontade para abandonar o cigarro.

Outro aspecto é que ali se constitui um espaço para a troca de experiências sobre o andamento da cessação, os sucessos alcançados e o enfrentamento dos sintomas de abstinência, os quais, conforme Dórea ${ }^{16}$, são fonte de grande sofrimento individual e um fator dificultador na decisão de manter o abandono:

Parei de fumar abruptamente! Quatro dias após a primeira sessão, mas ainda sinto vontade de fumar. (P8 S2)

Ainda não pensei no dia para parar de fumar. (P6 S2)

Parei de fumar há oito dias. (P6 S4)

Estou sentindo os sintomas da abstinência, sinto muita dor de cabę̧a e muita falta do cigarro. (P6 S4)

Os grupos significaram para os envolvidos uma fonte de "força" e apoio, também sendo confirmada a literatu- ra: Echer e Barreto ${ }^{14}$ apontam o acompanhamento profissional, além da utilização de medicamentos em algumas situações, como importantes fatores para o abandono. Sardinha ${ }^{17}$ encontrou relevância das técnicas cognitivocomportamentais em grupo para a cessação do hábito de fumar. Um estudo de revisão ${ }^{18}$ pontuou que intervenções farmacológicas (como a terapia de reposição de nicotina e a bupropiona) e psicossociais (como a terapia cognitivocomportamental e a intervenção motivacional face a face e por telefone) demonstram eficácia. Uma revisão sistemática da Cochrane Library apontou que o aconselhamento individual ou em grupo, assim como o tratamento farmacológico, aumentam a possibilidade de abandono do tabagismo ${ }^{19}$.

No entanto, ainda não há fornecimento adequado de medicamentos antitabaco na rede pública ${ }^{15}$. $\mathrm{O}$ atendimento profissional por pessoal capacitado e a estruturação em grupos de apoio também não são a rotina encontrada pelos usuários no sistema de saúde ${ }^{14}$. Além disso, o atendimento ainda hegemônico está calcado na busca de sinais e sintomas, ao invés da avaliação da pessoa como um todo e do seu contexto social ${ }^{11}$. Assim, ficam relegados a um segundo plano aspectos que influenciam no abandono ou não do tabagismo. Há necessidade de se ampliar o foco dos profissionais de saúde que enfrentam essa realidade ${ }^{20}$, pois a compreensão da representatividade que o tabaco tem para as pessoas e do contexto vivenciado por elas influencia o rompimento da dependência.

Um estudo realizado por Mattos et al. ${ }^{21}$ aponta que, durante a formação médica, os estudantes, apesar de entrarem em contato com o tema do tabagismo dentro de um currículo estruturado, não deixam a universidade com segurança para exercer a promoção à saúde no que tange à problemática do tabagismo. Isso já havia sido observado em um estudo britânico ${ }^{22}$, e, segundo o mesmo, tal fato ocorre por deficiência no treinamento dos estudantes. Percebe-se, assim, a necessidade da implementação de medidas para a mudança desse quadro. Com relação à experiência atual, acredita-se que tenha sido enriquecedora tanto para os graduandos em medicina como para os estudantes de enfermagem e odontologia. A troca de saberes ocorreu em todas as fases da pesquisa e propiciou um olhar diferenciado para o enfrentamento do problema do tabagismo em suas respectivas áreas de atuação. Além disso, a experiência também se constituiu em um estímulo aos estudantes para a iniciativa científica, que, apesar de estar preconizada nos diversos currículos, não é uma realidade frequente na graduação ${ }^{23}$.

Vale ressaltar que, a exemplo do que ocorre em muitos países desenvolvidos, a inserção da Medicina de Família e 
Comunidade nos currículos de graduação já é uma realidade, porém ainda é necessário consolidar, adequar, qualificar e expandir esta evolução ${ }^{24}$. Assim, a instituição de atividades de pesquisa científica nesse contexto, tais como o ocorrido na presente estratégia, só vem a sedimentar a importância da Atenção Primária como cenário de grande potencial gerador de conhecimento.

Deve-se, por fim, frisar a relevância da multiprofissionalidade. A participação multiprofissional foi defendida por Escher e Barreto ${ }^{14}$ no enfrentamento do tabaco e faz sentido quando se consideram as repercussões físicas sistêmicas advindas do seu uso. Torna clara para os participantes que há um interesse genuíno por parte de um grupo de profissionais no combate ao tabagismo, e que há de fato a disponibilidade de uma equipe de apoio nesse processo. Um questionamento para o futuro é se outros profissionais da área da saúde, tais como fisioterapeutas ou psicólogos, deveriam também fazer parte dos grupos.

\section{Considerações finais}

Este trabalho foi realizado por meio do convênio entre PET-Saúde e a UNIMONTES e demonstrou uma possibilidade de construção de conhecimento interdisciplinar a respeito do enfrentamento do tabagismo, com a articulação ensino-serviço.

A análise dos discursos de um conjunto de indivíduos submetidos a um mesmo contexto permitiu identificar opiniões e sentimentos ambíguos dos fumantes em relação ao cigarro. Foi possível também observar o benefício da instituição dos grupos antitabagismo, os quais, para os indivíduos, representaram um apoio durante a tentativa de abandono do tabaco. Conforme os relatos dos envolvidos, ficou evidente que essa alternativa de formação de grupos terapêuticos no cenário da Atenção Primária, com participação multiprofissional, associada ou não ao uso de medicamentos, é viável e pode resultar em êxito.

Por fim, salienta-se a necessidade de preparo e organização do sistema de saúde, assim como das instituições de ensino, para gerar uma abordagem mais completa à pessoa que fuma e para possibilitar a melhora do controle do tabagismo.

\section{Referências}

1. Ministério da Saúde (BR). Instituto Nacional do Câncer. Estimativas de incidência e mortalidade por câncer no Brasil. Rio de Janeiro: Ministério da Saúde; 2003
2. Mathers CD, Loncar D. Projections of global mortality and burden of desease from 2022 a 2030. PloS Med. 2006; 3(11): 442. http://dx.doi. org/10.1371/journal.pmed.0030442

3. Ministério da Saúde (BR). Portaria 1886/GM. Anexo 1 - Normas e diretrizes do Programa de Agentes Comunitários de Saúde - PACS. Brasília: Ministério da Saúde; 1997.

4. Wannmacher L. Tratamento medicamentoso antitabagismo. Uso Racional de Medicamentos: temas selecionados. 2007; 4(4): 1-6.

5. Oliveira MC. Os modelos de cuidados como eixo de estruturação de atividades interdisciplinares e multiprofissionais em saúde. Rev Bras Educ Méd. 2008; 32(3): 347-55. http://dx.doi.org/10.1590/S010055022008000300009

6. Thiollent M. Metodologia da pesquisa-ação. $12^{a}$ ed. São Paulo: Cortez; 2003.

7. Marques JB, Aprígio DP, Mello HLS, Silva JD, Pinto LN, Machado DCD, et al. Contribuições da equipe multiprofissional de saúde no programa saúde da família (PSF): uma atualização da literatura. Rev Baiana Saúde Pública. 2007; 31(2): 246-55.

8. Minayo MCS. O desafio do conhecimento. $5^{a}$ ed. São Paulo: Hucitec; 1998.

9. Ministério da Saúde (BR). Instituto Nacional do Câncer. Coordenação de prevenção e vigilância (CONPREV). Abordagem e tratamento do fumante. Rio de Janeiro: INCA; 2001.

10. Fagerström KO. Measuring degree of physical dependence to tobacco smoking with reference to individualization of treatment. Addict Behav. 1978; 3(3-4): 235-41. http://dx.doi.org/10.1016/03064603(78)90024-2

11. Borges MTT, Simões-Barbosa RH. Cigarro "companheiro": tabagismo feminino em uma abordagem crítica de gênero. Cad Saúde Pública. 2008; 24(12): 2834-42. http://dx.doi.org/10.1590/S0102$311 \times 2008001200012$

12. Malbergier A, Oliveira Júnior HP. Dependência de tabaco e comorbidade psiquiátrica. Rev Psiq Clin. 2005; 32(5): 276-82. http://dx.doi.org/10.1590/ S0101-60832005000500005

13. Focchi GR, Malbergier A, Ferreira MP. Tabagismo: dos fundamentos ao tratamento. São Paulo: Lemos; 2006.

14. Echer IC, Barreto SSM. Determinação e apoio como fatores de sucesso no abandono do tabagismo. Rev Latino-Am Enfermagem. 2008; 16(3): 445-51. http://dx.doi.org/10.1590/S0104-11692008000300018

15. Leitão Filho FS, Galduróz JCF, Noto AR, Nappo AS, Carlini EA, Nascimento $A O$, et al. Levantamento randomizado sobre a prevalência do tabagismo nos maiores municípios do Brasil. J Bras Pneumol. 2009; 35(12): 1204-11.

16. Dórea AJP, Botelho C. Fatores dificultadores da cessação do tabagismo. J Bras Pneumol. 2004; 30(2): 41-6.

17. Sardinha A, Oliva AD, D'augustin J, Ribeiro F, Falcone EMO. Intervenção cognitivo-comportamental com grupos para o abandono do cigarro. Rev Bras Ter Cogn. 2005; 1(1): 83-90.

18. Mazoni CG, Fernandes S, Pierozan OS, Moreira T, Freese L, Ferigolo $M$, et al. A eficácia das intervenções farmacológicas e psicossociais para o tratamento do tabagismo: revisão da literatura. Estud Psicol (Natal). 2008; 13(2): 133-40. http://dx.doi.org/10.1590/S1413294X2008000200005

19. Cahill K, Moher M, Lancaster T. Workplace interventions for smoking cessation. Cochrane Database of Systematic Reviews. 2008; (4): CD003440. http://dx.doi.org/10.1002/14651858.CD003440.pub3.

20. Hortense FTP, Carmagnani MIS, Brêtas ACP. O significado do tabagismo no contexto do câncer de laringe. Rev Bras Enferm. 2008; 61(1): 24-30. http://dx.doi.org/10.1590/S0034-71672008000100004

21. Mattos MHO, Silva LA, Franken RA. Tabagismo no currículo da faculdade de ciências médicas de São Paulo. Rev Bras Educ Med. 2009; 33(1): 33-9. http://dx.doi.org/10.1590/S0100-55022009000100005

22. Roddy E, Rubin P, Britton J. A study of smoking and smoking cessation on the curricula of UK medical schools. Tob Control. 2004; 13: 74-7. http://dx.doi.org/10.1136/tc.2003.004572

23. Oliveira NA, Alves LA, Luz MR. Iniciação científica na graduação: o que diz o estudante de medicina? Rev Bras Educ Med. 2008; 32(3): 309-14. http://dx.doi.org/10.1590/S0100-55022008000300005

24. Andreson MIP, Demarzo MMP, Rodrigues RD. A medicina de família e comunidade, a Atenção Primária à saúde e o ensino de graduação: recomendações e potencialidades. Rev bras med fam comunidade. 2007; 3(11): 157-72 